\title{
Asymptotic Stability and Exponential Stability of Impulsive Delayed Hopfield Neural Networks
}

\author{
Jing Chen, ${ }^{1}$ Xiaodi $\mathrm{Li}^{2,3}$ and Dequan Wang ${ }^{4}$ \\ ${ }^{1}$ Department of Mathematics, Shandong University, Jinan 250100, China \\ ${ }^{2}$ School of Mathematical Sciences, Shandong Normal University, Jinan 250014, China \\ ${ }^{3}$ Research Center on Logistics Optimization and Prediction of Engineering Technology, Jinan, Shandong 250014, China \\ ${ }^{4}$ School of Computer Science, Fudan University, Shanghai 200433, China
}

Correspondence should be addressed to Xiaodi Li; sodymath@163.com

Received 27 June 2013; Accepted 11 August 2013

Academic Editor: Jinde Cao

Copyright (C) 2013 Jing Chen et al. This is an open access article distributed under the Creative Commons Attribution License, which permits unrestricted use, distribution, and reproduction in any medium, provided the original work is properly cited.

A criterion for the uniform asymptotic stability of the equilibrium point of impulsive delayed Hopfield neural networks is presented by using Lyapunov functions and linear matrix inequality approach. The criterion is a less restrictive version of a recent result. By means of constructing the extended impulsive Halanay inequality, we also analyze the exponential stability of impulsive delayed Hopfield neural networks. Some new sufficient conditions ensuring exponential stability of the equilibrium point of impulsive delayed Hopfield neural networks are obtained. An example showing the effectiveness of the present criterion is given.

\section{Introduction}

In the last several years, Hopfield neural networks (HNN) have received especially considerable attention due to their extensive applications in solving optimization problem, traveling salesman problem, and many other subjects in recent years [1-9]. In hardware implementation of neural networks, time delays are inevitably present due to the finite switching speeds of the amplifiers. Hence, it is vital to investigate the stability of delayed HNN. Recently, various results for the stability of delayed HNN are obtained via different approaches. In [3], Rakkiyappan and Balasubramaniam studied the exponential stability for fuzzy impulsive neural networks by utilizing the Lyapunov-Krasovskii functional and the linear matrix inequality approach. In [8], Li studied the global robust stability for stochastic interval neural networks with continuously distributed delays of neutral type based on the similar methods. In [9], Xia et al. derived some sufficient conditions for the synchronization problem of coupled identical Yang-Yang type fuzzy cellular neural networks with timevarying delays based on using the invariance principle of functional differential equations.
On the other hand, impulsive differential equations have attracted a great deal of attention due to its potential applications in biological systems, chemical reactions, and various results are obtained; for instance, see [10-14]. Impulses can make unstable systems stable, and stable systems can become unstable after impulse effects. Hence, the stability properties of impulsive HNN with time delays have become an important topic of theoretical studies and have been investigated by many researchers; see [5, 6, 15-22]. In [5], Zhang and Sun obtained a result for the uniform stability of the equilibrium point of the impulsive $\mathrm{HNN}$ systems with time delays by using Lyapunov functions and analysis technique. In [6], global exponential stability of impulsive delay HNN is investigated by applying the piecewise continuous vector Lyapunov function.

The purpose of this paper is to present some sufficient conditions for uniform asymptotic stability and global exponential stability of impulsive $\mathrm{HNN}$ with time delays by means of constructing the extended impulsive Halanay inequality which is different from that given in [23], Lyapunov functional methods, and linear matrix inequality approach. 
The results here are also discussed from the point of view of thier comparison with the earlier results. Our results improve and generalize the earlier results. At last, we discuss an example to illustrate the advantage of the results we obtained.

\section{Systems Description and Preliminaries}

Let $\mathbb{R}$ denote the set of real numbers, let $\mathbb{R}_{+}$denote the set of nonnegative real numbers, and let $\mathbb{R}^{n}$ denote the $n$ dimensional real space equipped with the Euclidean norm $\|\cdot\|$.

Consider the following impulsive and delayed HNN model:

$$
\begin{aligned}
x_{i}^{\prime}(t)= & -c_{i} x_{i}(t)+\sum_{j=1}^{n} a_{i j} f_{j}\left(x_{j}(t)\right) \\
& +\sum_{j=1}^{n} b_{i j} g_{j}\left(x_{j}\left(t-\tau_{j}(t)\right)\right)+I_{i}, \quad t \neq t_{k}, \quad t \geq t_{0}, \\
\left.\Delta x_{i}\right|_{t=t_{k}} & =x_{i}\left(t_{k}\right)-x_{i}\left(t_{k}^{-}\right), \quad i \in \Lambda, k=1,2, \ldots,
\end{aligned}
$$

where $\Lambda=\{1,2, \ldots, n\} . n \geq 2$ corresponds to the number of units in a neural network; the impulse times $t_{k}$ satisfy $0 \leq t_{0}<t_{1}<\cdots<t_{k}<\cdots, \lim _{k \rightarrow+\infty} t_{k}=+\infty ; x_{i}$ corresponds to the membrane potential of the unit $i$ at time $t$; $c_{i}$ is positive constant; $f_{j}, g_{j}$ denote, respectively, the measures of response or activation to its incoming potentials of the unit $j$ at time $t$ and $t-\tau_{j}(t)$; constant $a_{i j}$ denotes the synaptic connection weight of the unit $j$ on the unit $i$ at time $t$; constant $b_{i j}$ denotes the synaptic connection weight of the unit $j$ on the unit $i$ at time $t-\tau_{j}(t) ; I_{i}$ is the input of the unit $i ; \tau_{j}(t)$ is the transmission delay of the $j$ th neuron such that $0<\tau_{j}(t) \leq$ $\tau, t \geq t_{0}$, and $j \in \Lambda$.

Assume that system (1) is supplemented with initial conditions of the form

$$
x(s)=\phi(s), \quad s \in\left[t_{0}-\tau, t_{0}\right],
$$

where $x(s)=\left(x_{1}(s), x_{2}(s), \ldots, x_{n}(s)\right)^{T}, \phi(s)=\left(\phi_{1}(s), \phi_{2}(s)\right.$, $\left.\ldots, \phi_{n}(s)\right)^{T} \in P C\left(\left[t_{0}-\tau, t_{0}\right], \mathbb{R}^{n}\right)$, and $P C\left(I, \mathbb{R}^{n}\right)=\{\psi: I \rightarrow$ $\mathbb{R}^{n}$, which is continuous everywhere except at finite number of points $t_{k}$, at which $\psi\left(t_{k}^{+}\right)$and $\psi\left(t_{k}^{-}\right)$exist and $\left.\psi\left(t_{k}^{+}\right)=\psi\left(t_{k}\right)\right\}$. For any given $t \geq t_{0}, \psi \in P C\left([t-\tau, t], \mathbb{R}^{n}\right)$, the norm of $\psi$ is defined by $\|\psi\|_{\tau}=\sup _{t-\tau \leq \theta \leq t}|\psi(\theta)|$. For any $\sigma \geq 0$, let $P_{\delta}(\sigma)=\left\{\psi \in P C\left([\sigma-\tau, \sigma], \mathbb{R}^{n}\right):\|\psi\|<\delta\right\}$.

In this paper, we assume that some conditions are satisfied, so that the equilibrium point of (1) without impulse does exist denoted by $x^{*}=\left(x_{1}^{*}, x_{2}^{*}, \ldots, x_{n}^{*}\right)^{T}$; see $[2,5]$. Impulsive operator is viewed as perturbation of the equilibrium point of system (1) without impulsive effects. We assume that $\left.\Delta x_{i}\right|_{t=t_{k}}=x_{i}\left(t_{k}\right)-x_{i}\left(t_{k}^{-}\right)=d_{k}^{(i)}\left(x_{i}\left(t_{k}^{-}\right)-x^{*}\right), d_{k}^{(i)} \in \mathbb{R}$, and $i \in \Lambda, k=1,2, \ldots$
Since $x^{*}$ is an equilibrium point of (1), one can derive from (1) that the transformation $y_{i}=x_{i}-x_{i}^{*}, i \in \Lambda$, transforms system (1) into the following system:

$$
\begin{aligned}
y_{i}^{\prime}(t)= & -c_{i} y_{i}(t)+\sum_{j=1}^{n} a_{i j} \Omega_{j}\left(y_{j}(t)\right) \\
& +\sum_{j=1}^{n} b_{i j} \Gamma_{j}\left(y_{j}\left(t-\tau_{j}(t)\right)\right), \quad t \neq t_{k}, t \geq t_{0}, \\
y_{i}\left(t_{k}\right)= & \left(1+d_{k}^{(i)}\right) y_{i}\left(t_{k}^{-}\right), \quad i \in \Lambda, k=1,2, \ldots,
\end{aligned}
$$

where $\Omega_{j}\left(y_{j}(t)\right)=f_{j}\left(x_{j}^{*}+y_{j}(t)\right)-f_{j}\left(x_{j}^{*}\right), \Gamma_{j}\left(y_{j}(t)\right)=g_{j}\left(x_{j}^{*}+\right.$ $\left.y_{j}\left(t-\tau_{j}(t)\right)\right)-g_{j}\left(x_{j}^{*}\right)$.

Clearly, $x^{*}$ is uniformly asymptotically stable for system (1) if and only if the trivial solution of system (3) is uniformly asymptotically stable. Hence, we only need to prove the stability of the trivial solution of system (3).

Remark 1. If $x_{i}\left(t_{k}\right)=d_{k}^{(i)} x_{i}\left(t_{k}^{-}\right)$, then we cannot get $y_{i}\left(t_{k}\right)=$ $d_{k}^{(i)} y_{i}\left(t_{k}^{-}\right)$through the transformation $y_{i}=x_{i}-x_{i}^{*}$. So some of the results [5] are incorrect.

The following notations will be used throughout the paper. The notation $A^{T}$ and $A^{-1}$ means the transpose of and the inverse of a square matrix $A$. Let $y(t)=\left(y_{1}(t), y_{2}(t), \ldots\right.$, $\left.y_{n}(t)\right)^{T}, y_{\tau}=\left(y_{1}\left(t-\tau_{1}(t)\right), y_{2}\left(t-\tau_{2}(t)\right), \ldots, y_{n}\left(t-\tau_{n}(t)\right)\right)^{T} ; C=$ $\operatorname{diag}\left[-c_{1},-c_{2}, \ldots,-c_{n}\right]^{T}, A=\left(a_{i j}\right)_{n \times n}, B=\left(b_{i j}\right)_{n \times n}, D_{k}=$ $\operatorname{diag}\left[1+d_{k}^{(1)}, 1+d_{k}^{(2)}, \ldots, 1+d_{k}^{(n)}\right]^{T} ; \Omega(y)=\left[\Omega_{1}\left(y_{1}\right)\right.$, $\left.\Omega_{2}\left(y_{2}\right), \ldots, \Omega_{n}\left(y_{n}\right)\right]^{T}, \Gamma\left(y_{\tau}\right)=\left[\Gamma_{1}\left(y_{1}\left(t-\tau_{1}(t)\right)\right), \Gamma_{2}\left(y_{2}(t-\right.\right.$ $\left.\left.\left.\tau_{2}(t)\right)\right), \ldots, \Gamma_{n}\left(y_{n}\left(t-\tau_{n}(t)\right)\right)\right]^{T}$. Then system (3) with initial condition becomes

$$
\begin{gathered}
y^{\prime}(t)=C y(t)+A \Omega(y(t))+B \Gamma\left(y_{\tau}\right), \quad t \neq t_{k}, t \geq t_{0}, \\
y\left(t_{k}\right)=D_{k} y\left(t_{k}^{-}\right), \quad k=1,2, \ldots, \\
y\left(t_{0}+\theta\right)=\varphi(\theta), \quad \theta \in[-\tau, 0]
\end{gathered}
$$

where $\varphi(\theta)=x\left(t_{0}+\theta\right)-x^{*}$.

We introduce some definitions as follows.

Definition 2 (see [10]). The function $V(t, s):[0, \infty) \times \mathbb{R}^{n} \rightarrow$ $\mathbb{R}_{+}$belongs to class $v_{0}$ if

$$
\begin{aligned}
& \left(A_{1}\right) V \text { is continuous on each of the sets }\left[t_{k-1}, t_{k}\right) \times \mathbb{R}^{n} \text { and } \\
& \lim _{(t, z) \rightarrow\left(t_{k}^{-}, s\right)} V(t, z)=V\left(t_{k}^{-}, s\right) \text { exists; } \\
& \left(A_{2}\right) V(t, s) \text { is locally Lipschitzian in } s \text { and } V(t, 0) \equiv 0 .
\end{aligned}
$$

Definition 3 (see [10]). Let $V \in v_{0}$, for any $(t, s) \in\left[t_{k-1}, t_{k}\right) \times$ $\mathbb{R}^{n}$; the upper right-hand Dini derivative of $V(t, s)$ along the solution of (4) is defined by

$$
\begin{aligned}
D^{+} V(t, \psi(0)) \\
\quad=\frac{\limsup _{h \rightarrow 0^{+}}\{V(t+h, y(t, \psi)(t+h))-V(t, \psi(0))\}}{h} .
\end{aligned}
$$


Definition 4 (see [11]). Assume that $y(t)=y(\sigma, \varphi)(t)$ is the solution of (4) through $(\sigma, \varphi)$. Then the zero solution of (4) is said to be

(1) uniformly stable, if for any $\sigma \geq t_{0}$ and $\varepsilon>0$, there exists a $\delta=\delta(\varepsilon)>0$ such that $\varphi \in P C_{\delta}(\sigma)$ implies that $\|y(\sigma, \varphi)(t)\|<\varepsilon, t \geq \sigma ;$

(2) uniformly asymptotically stable, if it is uniformly stable, and there exists a $\delta>0$ such that for any $\varepsilon>0$, $\sigma \geq t_{0}$, there is a $T=T(\varepsilon)>0$ such that $\varphi \in P C_{\delta}(\sigma)$ implies that $\|y(\sigma, \varphi)(t)\|<\varepsilon, t \geq \sigma+T$;

(3) globally exponentially stable, if for any $\varphi \in P C([-\tau$, $\left.0], \mathbb{R}^{n}\right)$, there exist constants $M, \mu>0$ such that

$$
\|y(t)\|<M\|\varphi\|_{\tau} e^{-\mu(t-\sigma)}, \quad t \geq \sigma .
$$

In this paper, we always assume that the following assumption holds:

$\left(H_{0}\right)$ there exist constants $M, N>0$ such that

$$
\Omega^{T}(y) \Omega(y) \leq M y^{T} y, \quad \Gamma^{T}\left(y_{\tau}\right) \Gamma\left(y_{\tau}\right) \leq N y_{\tau}^{T} y_{\tau} .
$$

In addition, we have the following basic lemmas.

Lemma 5 (see [24]). For any vectors $a, b \in \mathbb{R}^{n}$, the inequality

$$
\pm 2 a^{T} b \leq a^{T} X a+b^{T} X^{-1} b
$$

holds, in which $X$ is any $n \times n$ matrix with $X>0$.

Lemma 6 (see [25]). Assume that there exist constants $P, Q>$ 0 and $m(t) \in P C\left(\left[t_{0}-\tau, \infty\right), \mathbb{R}_{+}\right)$such that

(i) for $t=t_{k}, m\left(t_{k}\right) \leq \gamma_{k} m\left(t_{k}^{-}\right), \gamma_{k}>0$ are constants and satisfy $\max _{k \in \mathbb{Z}_{+}}\left\{1 / \gamma_{k}, 1\right\}<P / Q$;

(ii) for $t \geq t_{0}, t \neq t_{k}$,

$$
D^{+} m(t) \leq-P m(t)+Q \widetilde{m}(t),
$$

where $\widetilde{m}(t)=\sup _{t-\tau \leq s \leq t} m(s)$.

Then for $t \geq t_{0}$,

$$
m(t) \leq \widetilde{m}\left(t_{0}\right)\left(\prod_{t_{0}<t_{k} \leq t} \gamma_{k}\right) e^{-\lambda\left(t-t_{0}\right)},
$$

where $\lambda$ satisfies the following inequality:

$$
0<\lambda \leq P-Q \max _{k \in \mathbb{Z}_{+}}\left\{\frac{1}{\gamma_{k}}, 1\right\} \cdot e^{\lambda \tau} \text {. }
$$

\section{Main Results}

In this section, we will establish some theorems which provide sufficient conditions for uniformly asymptotically stable and global exponential stability of system (1).

Theorem 7. The equilibrium point the system (1) is uniformly asymptotically stable, if there exists $n \times n$ symmetric, and positive definite matrix $P$ satisfies the following conditions:
$\left(H_{1}\right) \bar{\eta} \doteq \prod_{k=1}^{\infty} \max \left\{\eta_{k}, 1\right\}<\infty$, where $\eta_{k}$ is the largest eigenvalue of $P^{-1} D_{k} P D_{k}$;

$\left(H_{2}\right) \lambda_{3}<(-M-N) / \lambda_{1}$, where $\lambda_{1}$ is the smallest eigenvalue of $P$ and $\lambda_{3}$ is the largest eigenvalue of $P^{-1}(C P+P C+$ $\left.P A A^{T} P+P B B^{T} P\right)$.

Proof. First, we will prove that the zero solution of system (4) is uniformly stable. For any $\varepsilon>0$, we may choose a $\delta>0$ such that $\delta \leq \sqrt{\left(\lambda_{1} / \bar{\eta} \lambda_{2}\right) \varepsilon}$, where $\lambda_{2}$ is the largest eigenvalue of $P$. For any $\sigma \geq t_{0}, \varphi \in P C_{\delta}(\sigma)$, let $y(t)=y(\sigma, \varphi)(t)$ be a solution of (4) through $(\sigma, \varphi), \sigma \geq t_{0}$ (for convenience, that we assume $\left.\sigma=t_{0}\right)$; then we can prove that $\|y(t)\|<\varepsilon, t \geq t_{0}$.

Consider the following Lyapunov function: $V(t, y(t))=$ $y^{T}(t) P y(t)$; then we have

$$
\lambda_{1}\|y(t)\|^{2} \leq V(t, y(t)) \leq \lambda_{2}\|y(t)\|^{2} .
$$

By virtue of Lemma 5 , we obtain for $t \in\left[t_{k}, t_{k+1}\right), k=1,2, \ldots$,

$$
\begin{aligned}
D^{+} V( & t, y(t))\left.\right|_{(4)} \\
= & \left(y^{T}(t)\right)^{\prime} P y(t)+y^{T}(t) P y^{\prime}(t) \\
= & \left(C y(t)+A \Omega(y(t))+B \Gamma\left(y_{\tau}\right)\right)^{T} P y(t) \\
& +y^{T}(t) P\left(C y(t)+A \Omega(y(t))+B \Gamma\left(y_{\tau}\right)\right) \\
= & y^{T}(t) C P y(t)+\Omega^{T}(y(t)) A^{T} P y(t) \\
& +\Gamma^{T}\left(y_{\tau}\right) B^{T} P y(t)+y^{T}(t) P C y(t) \\
& +y^{T}(t) P A \Omega(y(t))+y^{T}(t) P B \Gamma\left(y_{\tau}\right) \\
= & y^{T}(t)(C P+P C) y(t) \\
& +2 \Omega^{T}(y(t)) A^{T} P y(t)+2 \Gamma^{T}\left(y_{\tau}\right) B^{T} P y(t) \\
\leq & y^{T}(t)(C P+P C) y(t) \\
& +\Omega^{T}(y(t)) \Omega(y(t))+y^{T}(t) P A A^{T} P y(t) \\
& +\Gamma^{T}\left(y_{\tau}\right) \Gamma\left(y_{\tau}\right)+y^{T}(t) P B B^{T} P y(t) \\
\leq & y^{T}(t)\left(C P+P C+P A A^{T} P+P B B^{T} P\right) y(t) \\
& +\lambda_{3} y^{T}(t) P y(t)+M y^{T}(t) y(t)+N y_{\tau}^{T} y_{\tau} \\
\leq & \lambda_{3} y^{T}(t) P y(t)+M \lambda_{1}^{-1} y^{T}(t) P y(t) \\
& +M \lambda_{1}^{-1} y_{\tau}^{T} P y_{\tau} \\
& \left.\lambda_{3}\right] y^{T}(t) P y(t)+N \lambda_{1}^{-1} y_{\tau}^{T} P y_{\tau} .
\end{aligned}
$$

First, it is obvious that for $t_{0}-\tau \leq t \leq t_{0}$,

$$
\lambda_{1}\|y(t)\|^{2} \leq V(t, y(t)) \leq \lambda_{2} \delta^{2} \leq \bar{\eta}^{-1} \lambda_{1} \varepsilon^{2} .
$$

Then we can prove that for $t \in\left[t_{0}, t_{1}\right)$,

$$
V(t, y(t)) \leq \bar{\eta}^{-1} \lambda_{1} \varepsilon^{2} .
$$


Suppose that this is not true; then there exists $\widehat{t} \in\left[t_{0}, t_{1}\right)$ such that $V(\widehat{t}, y(\widehat{t}))>\bar{\eta}^{-1} \lambda_{1} \varepsilon^{2}$.

Set

$$
\check{t}=\sup \left\{t \mid s \in\left[t_{0}, t\right), V(s, y(s)) \leq \bar{\eta}^{-1} \lambda_{1} \varepsilon^{2}\right\} .
$$

It is obvious that $\check{t}<\widehat{t}$. Then it follows that

$$
\begin{aligned}
&\left(I_{a}\right) V(t, y(t)) \leq \bar{\eta}^{-1} \lambda_{1} \varepsilon^{2}, t \in\left[t_{0}, \check{t}\right) ; \\
&\left(I I_{a}\right) V(\check{t}, y(\check{t}))=\bar{\eta}^{-1} \lambda_{1} \varepsilon^{2} ; \\
&\left(I I I_{a}\right) \text { for any } \delta>0, \text { there exists } t_{\delta} \in(\check{t}, \check{t}+\delta) \text { such that } \\
& V\left(t_{\delta}, y\left(t_{\delta}\right)\right)>\bar{\eta}^{-1} \lambda_{1} \varepsilon^{2} .
\end{aligned}
$$

So

$$
V(\check{t}, y(\check{t}))=\bar{\eta}^{-1} \lambda_{1} \varepsilon^{2} \geq V\left(t, y_{\tau}\right), \quad \check{t}-\tau \leq t \leq \check{t} .
$$

In view of condition $\left(\mathrm{H}_{2}\right)$, from (13), we obtain

$$
\begin{aligned}
D^{+} V(\check{t}, y(\check{t})) & \leq\left[\lambda_{3}+M \lambda_{1}^{-1}\right] y^{T}(\check{t}) P y(\check{t})+N \lambda_{1}^{-1} y_{\tau}^{T} P y_{\tau} \\
& \leq\left[\lambda_{3}+M \lambda_{1}^{-1}+N \lambda_{1}^{-1}\right] V(\check{t}, y(\check{t})) \\
& <0,
\end{aligned}
$$

which is a contradiction with $\left(I I I_{a}\right)$. Hence, (15) holds. Considering

$$
\begin{aligned}
V\left(t_{1}, y\left(t_{1}\right)\right) & =y^{T}\left(t_{1}\right) P y\left(t_{1}\right) \\
& =y^{T}\left(t_{1}^{-}\right) D_{1} P D_{1} y\left(t_{1}^{-}\right) \\
& \leq \eta_{1} y^{T}\left(t_{1}^{-}\right) P y\left(t_{1}^{-}\right) \\
& =\eta_{1} V\left(t_{1}^{-}, y\left(t_{1}^{-}\right)\right) \\
& \leq \eta_{1} \bar{\eta}^{-1} \lambda_{1} \varepsilon^{2} \\
& \leq \max \left\{\eta_{1}, 1\right\} \bar{\eta}^{-1} \lambda_{1} \varepsilon^{2},
\end{aligned}
$$

we will prove that for $t \in\left[t_{1}, t_{2}\right)$,

$$
V(t, y(t)) \leq \max \left\{\eta_{1}, 1\right\} \bar{\eta}^{-1} \lambda_{1} \varepsilon^{2} .
$$

Suppose that this is false; then we can define

$$
\vec{t}=\sup \left\{t \mid s \in\left[t_{1}, t\right), V(s, y(s)) \leq \max \left\{\eta_{1}, 1\right\} \bar{\eta}^{-1} \lambda_{1} \varepsilon^{2}\right\} .
$$

Similarly, we can obtain

$$
\left(I_{b}\right) V(t, y(t)) \leq \max \left\{\eta_{1}, 1\right\} \bar{\eta}^{-1} \lambda_{1} \varepsilon^{2}, t \in\left[t_{1}, \vec{t}\right) ;
$$$$
\left(I I_{b}\right) V(\vec{t}, y(\vec{t}))=\max \left\{\eta_{1}, 1\right\} \bar{\eta}^{-1} \lambda_{1} \varepsilon^{2} ;
$$

$\left(I I I_{b}\right)$ for any $\delta>0$, there exists $t_{\delta} \in(\vec{t}, \vec{t}+\delta)$ such that $V\left(t_{\delta}, y\left(t_{\delta}\right)\right)>\max \left\{\eta_{1}, 1\right\} \bar{\eta}^{-1} \lambda_{1} \varepsilon^{2}$

So

$$
\begin{array}{r}
V(\vec{t}, y(\vec{t}))=\max \left\{\eta_{1}, 1\right\} \bar{\eta}^{-1} \lambda_{1} \varepsilon^{2} \geq V\left(t, y_{\tau}\right), \\
\vec{t}-\tau \leq t \leq \vec{t} .
\end{array}
$$

In fact, if $\vec{t}-\tau \geq t_{1}$, then it is obvious that inequality (22) holds. If $\vec{t}-\tau<t_{1}$, then $V\left(t, y_{\tau}\right) \leq \bar{\eta}^{-1} \lambda_{1} \varepsilon^{2} \leq \max \left\{\eta_{1}, 1\right\} \bar{\eta}^{-1} \lambda_{1} \varepsilon^{2}=$ $V(\vec{t}, y(\vec{t}))$. So, inequality (22) still holds.

Considering condition $\left(\mathrm{H}_{2}\right)$, from (13), we obtain

$$
\begin{aligned}
D^{+} V(\vec{t}, y(\vec{t})) & \leq\left[\lambda_{3}+M \lambda_{1}^{-1}\right] y^{T}(\vec{t}) P y(\vec{t})+N \lambda_{1}^{-1} y_{\tau}^{T} P y_{\tau} \\
& \leq\left[\lambda_{3}+M \lambda_{1}^{-1}+N \lambda_{1}^{-1}\right] V(\vec{t}, y(\vec{t})) \\
& <0,
\end{aligned}
$$

which contradicts $\left(I I I_{b}\right)$. Hence, (20) holds.

By induction hypothesis, we may prove, in general, that for $t \in\left[t_{m}, t_{m+1}\right)$,

$$
V(t, y(t)) \leq \prod_{k=1}^{m} \max \left\{\eta_{k}, 1\right\} \bar{\eta}^{-1} \lambda_{1} \varepsilon^{2}
$$

that is,

$$
V(t, y(t)) \leq \prod_{t_{0}<t_{k} \leq t} \max \left\{\eta_{k}, 1\right\} \bar{\eta}^{-1} \lambda_{1} \varepsilon^{2} .
$$

Finally, we arrive at

$$
\lambda_{1}\|y(t)\|^{2} \leq V(t, y(t)) \leq \lambda_{1} \varepsilon^{2}, \quad t \geq t_{0} .
$$

Therefore, we obtain $\|y(t)\|<\varepsilon, t \geq t_{0}$. In view of the choice of $\delta$, the zero solution of (4) is uniformly stable; that is, the equilibrium point of (1) is uniformly stable.

Next we show the uniformly asymptotical stability. For any given $G>0$, we find a corresponding $\delta>0$ such that for any $\varphi \in P C_{\delta}(\sigma)$ implies that $\|y(t)\|=\left\|y\left(t_{0}, \varphi\right)(t)\right\| \leq G$, $t \geq \sigma=t_{0}$; that is, $V(t, y(t)) \leq \lambda_{2} G^{2}$.

For any small $\varepsilon \in(0, G)$, we choose $\vec{N}=\vec{N}(\varepsilon) \in \mathbb{Z}_{+}$such that

$$
\begin{aligned}
\sqrt{\frac{\vec{N} \lambda_{1}}{2 \lambda_{2}}} \varepsilon & <G \leq \sqrt{\frac{(\vec{N}+1) \lambda_{1}}{2 \lambda_{2}}} \varepsilon, \\
\vec{N} & >\frac{-N}{\lambda_{3} \lambda_{1}+M+N} .
\end{aligned}
$$

In fact, it is feasible to choose small enough $\varepsilon \in(0, G)$ such that $\vec{N}$ in (27) is large enough to satisfy (28).

Since $\bar{\eta} \doteq \prod_{k=1}^{\infty} \max \left\{\eta_{k}, 1\right\}<\infty$ implies that $\sum_{k=1}^{\infty}\left(\max \left\{\eta_{k}, 1\right\}-1\right)<\infty$, there exists sufficient large $N^{\star} \epsilon$ $\mathbb{Z}_{+}$such that

$$
\begin{gathered}
\sum_{i=N^{\star}}^{\infty}\left(\max \left\{\eta_{i}, 1\right\}-1\right) \leq \frac{\lambda_{1} \varepsilon^{2}}{6 \lambda_{2} G^{2}}, \\
\max \left\{\eta_{k}, 1\right\}<\frac{1}{6 \vec{N}}+1, \quad k \geq N^{\star} .
\end{gathered}
$$

Let

$$
w_{1}=\frac{\left[1+\sum_{k=1}^{\infty}\left(\max \left\{\eta_{k}, 1\right\}-1\right)\right] \lambda_{2} G^{2}}{\left|\left[\lambda_{1} \lambda_{3}+M+N\right] \vec{N}+N\right|} \cdot \frac{2}{\varepsilon^{2}}+1 .
$$


Next we show that there exists $T_{1} \in\left[N^{\star}, N^{\star}+w_{1}\right]$ such that

$$
V\left(T_{1}, y\left(T_{1}\right)\right)<\frac{\lambda_{1} \varepsilon^{2}}{2} \vec{N} .
$$

Or else, for all $t \in\left[N^{\star}, N^{\star}+w_{1}\right]$,

$$
V(t, y(t)) \geq \frac{\lambda_{1} \varepsilon^{2}}{2} \vec{N} .
$$

Thus, we get

$$
\begin{array}{r}
V(t, y(t))+\frac{\lambda_{1} \varepsilon^{2}}{2} \geq \frac{\lambda_{1} \varepsilon^{2}}{2}(\vec{N}+1) \geq \lambda_{2} G^{2}>V\left(\xi, y_{\tau}\right), \\
t-\tau \leq \xi \leq t .
\end{array}
$$

From (13), we have

$$
\begin{aligned}
\left.D^{+} V(t, y(t))\right|_{(4)} \leq & {\left[\lambda_{3}+M \lambda_{1}^{-1}\right] y^{T}(t) P y(t) } \\
& +N \lambda_{1}^{-1} y_{\tau}^{T} P y_{\tau} \\
\leq & {\left[\lambda_{3}+M \lambda_{1}^{-1}\right] V(t, y(t)) } \\
& +N \lambda_{1}^{-1}\left(V(t, y(t))+\frac{\lambda_{1} \varepsilon^{2}}{2}\right) \\
\leq & {\left[\lambda_{3}+M \lambda_{1}^{-1}+N \lambda_{1}^{-1}\right] V(t, y(t))+\frac{\varepsilon^{2}}{2} N } \\
\leq & {\left[\lambda_{3}+M \lambda_{1}^{-1}+N \lambda_{1}^{-1}\right] \frac{\lambda_{1} \varepsilon^{2}}{2} \vec{N}+\frac{\varepsilon^{2}}{2} N } \\
\leq & \left\{\left[\lambda_{3} \lambda_{1}+M+N\right] \vec{N}+N\right\} \frac{\varepsilon^{2}}{2} .
\end{aligned}
$$

Integrating the above inequality from $N^{\star}$ to $N^{\star}+w_{1}$, we have

$$
\begin{aligned}
V\left(N^{\star}+\right. & \left.w_{1}, y\left(N^{\star}+w_{1}\right)\right) \\
\leq & V\left(N^{\star}, y\left(N^{\star}\right)\right)+\left\{\left[\lambda_{3} \lambda_{1}+M+N\right] \vec{N}+N\right\} \frac{\varepsilon^{2}}{2} w_{1} \\
& +\sum_{N^{\star}<t_{k}<N^{\star}+w_{1}}\left[V\left(t_{k}\right)-V\left(t_{k}^{-}\right)\right] \\
\leq & V\left(N^{\star}, y\left(N^{\star}\right)\right)+\left\{\left[\lambda_{3} \lambda_{1}+M+N\right] \vec{N}+N\right\} \frac{\varepsilon^{2}}{2} w_{1} \\
& +\sum_{N^{\star}<t_{k}<N^{\star}+w_{1}}\left[\max \left\{\eta_{k}, 1\right\}-1\right] V\left(t_{k}^{-}\right) \\
\leq & \lambda_{2} G^{2}+\left\{\left[\lambda_{3} \lambda_{1}+M+N\right] \vec{N}+N\right\} \frac{\varepsilon^{2}}{2} w_{1} \\
& +\sum_{N^{\star}<t_{k}<N^{\star}+w_{1}}\left[\max \left\{\eta_{k}, 1\right\}-1\right] \lambda_{2} G^{2}
\end{aligned}
$$

$$
\begin{aligned}
& \leq\left\{\left[\lambda_{3} \lambda_{1}+M+N\right] \vec{N}+N\right\} \frac{\varepsilon^{2}}{2} w_{1} \\
&+\left\{1+\sum_{N^{\star}<t_{k}<N^{\star}+w_{1}}\left[\max \left\{\eta_{k}, 1\right\}-1\right]\right\} \lambda_{2} G^{2} \\
&<0,
\end{aligned}
$$

which is a contradiction. So (31) holds. We may choose $T_{1}=$ $N^{\star}+w_{1}$.

We next claim that for all $t>T_{1}$,

$$
V(t, y(t))<\left(\frac{\vec{N}}{2}+\frac{1}{4}\right) \lambda_{1} \varepsilon^{2} .
$$

Suppose that this is not true; then there exists a $\tau_{2}>T_{1}$ such that

$$
V\left(\tau_{2}, y\left(\tau_{2}\right)\right) \geq\left(\frac{\vec{N}}{2}+\frac{1}{4}\right) \lambda_{1} \varepsilon^{2},
$$

and for $T_{1}<t<\tau_{2}$,

$$
V(t, y(t))<\left(\frac{\vec{N}}{2}+\frac{1}{4}\right) \lambda_{1} \varepsilon^{2} .
$$

Suppose that $T_{1} \in\left[t_{m}, t_{m+1}\right), m \in \mathbb{Z}_{+}$. We claim that $\tau_{2} \geq$ $t_{m+1}$. Otherwise, $\tau_{2} \in\left[T_{1}, t_{m+1}\right)$. Since (31) holds, it is clear that there exists a $\tau_{1} \in\left[T_{1}, \tau_{2}\right)$ such that

$$
\begin{gathered}
V\left(\tau_{1}, y\left(\tau_{1}\right)\right)=\frac{\vec{N}}{2} \lambda_{1} \varepsilon^{2}, \\
V\left(\tau_{1}, y\left(\tau_{1}\right)\right) \leq V(t, x(t)) \leq V\left(\tau_{2}, x\left(\tau_{2}\right)\right), \\
\tau_{1} \leq t \leq \tau_{2} .
\end{gathered}
$$

Furthermore, we note that

$$
\begin{array}{r}
V(t, y(t))+\frac{\lambda_{1} \varepsilon^{2}}{2} \geq \frac{\lambda_{1} \varepsilon^{2}}{2}(\vec{N}+1) \geq \lambda_{2} G^{2}>V\left(\xi, y_{\tau}\right), \\
t-\tau \leq \xi \leq t .
\end{array}
$$

From (13), we have

$$
\begin{array}{r}
D^{+} V(t, y(t)) \leq\left\{\left[\lambda_{3} \lambda_{1}+M+N\right] \vec{N}+N\right\} \frac{\varepsilon^{2}}{2}<0, \\
\tau_{1} \leq t \leq \tau_{2},
\end{array}
$$

which implies that

$$
V\left(\tau_{2}, y\left(\tau_{2}\right)\right) \leq V\left(\tau_{1}, y\left(\tau_{1}\right)\right) .
$$

This is a contradiction.

Hence, we obtain $\tau_{2} \geq t_{m+1}$; without loss of generality, we may suppose that $\tau_{2} \in\left[t_{m+q}, t_{m+q+1}\right), q \geq 1$. Next we first claim that there exists $\tau_{1}>0$ satisfying $\tau_{2}>\tau_{1}>T_{1}$ such that

$$
V\left(\tau_{1}, y\left(\tau_{1}\right)\right) \geq \frac{\vec{N}}{2} \lambda_{1} \varepsilon^{2} .
$$


Suppose that this is false; then for all $t \in\left(T_{1}, \tau_{2}\right)$,

$$
V(t, y(t))<\frac{\vec{N}}{2} \lambda_{1} \varepsilon^{2},
$$

which implies that $\tau_{2}=t_{m+q}$ in view of (37). Consequently, we have

$$
\begin{gathered}
V\left(t_{m+q}, y\left(t_{m+q}\right)\right) \geq\left(\frac{\vec{N}}{2}+\frac{1}{4}\right) \lambda_{1} \varepsilon^{2}, \\
V\left(t_{m+q}^{-}, y\left(t_{m+q}^{-}\right)\right)<\frac{\vec{N}}{2} \lambda_{1} \varepsilon^{2},
\end{gathered}
$$

which implies that

$$
\begin{aligned}
& \left(\max \left\{\eta_{m+q}, 1\right\}-1\right) \lambda_{2} G^{2} \\
& \quad \geq\left(\max \left\{\eta_{m+q}, 1\right\}-1\right) V\left(t_{m+q}^{-}, y\left(t_{m+q}^{-}\right)\right)>\frac{1}{4} \lambda_{1} \varepsilon^{2} .
\end{aligned}
$$

Hence, we get $\max \left\{\eta_{m+q}, 1\right\}>1+\left(\lambda_{1} \varepsilon^{2} / 4 \lambda_{2} G^{2}\right)$, which contradicts (29). So (43) holds.

Therefore, there are two situations $\tau_{2}>t_{m+q}$ and $\tau_{2}=$ $t_{m+q}$. Next we discuss them, respectively.

$$
\begin{gathered}
\left(b_{1}\right) \text { If } \tau_{2}>t_{m+q} \text {, let } \\
\widetilde{\tau_{1}}=\inf _{\tau_{1} \leq t<\tau_{2}}\left\{t \mid s \in\left[t, \tau_{2}\right], \frac{\vec{N}}{2} \lambda_{1} \varepsilon^{2} \leq V(s, y(s))\right. \\
\left.<V\left(\tau_{2}, y\left(\tau_{2}\right)\right)\right\} .
\end{gathered}
$$

We first show that $\widetilde{\tau_{1}}<t_{m+q}$. Suppose on the contrary that $\widetilde{\tau_{1}} \in\left[t_{m+q}, \tau_{2}\right) ;$ then

$$
\begin{aligned}
& V(t, y(t))+\frac{\lambda_{1} \varepsilon^{2}}{2} \geq \frac{\lambda_{1} \varepsilon^{2}}{2}(\vec{N}+1) \\
& \geq \lambda_{2} G^{2}>V\left(\xi, y_{\tau}\right), \\
& t-\tau \leq \xi \leq t, \quad t \in\left[\widetilde{\tau_{1}}, \tau_{2}\right] .
\end{aligned}
$$

From (13), we have

$$
\begin{array}{r}
D^{+} V(t, y(t)) \leq\left\{\left[\lambda_{3} \lambda_{1}+M+N\right] \vec{N}+N\right\} \frac{\varepsilon^{2}}{2}<0, \\
\widetilde{\tau_{1}} \leq t \leq \tau_{2},
\end{array}
$$

which implies that

$$
V\left(\tau_{2}, y\left(\tau_{2}\right)\right) \leq V\left(\widetilde{\tau_{1}}, y\left(\widetilde{\tau_{1}}\right)\right)
$$

which is a contradiction with the definition of $\widetilde{\tau_{1}}$. Thus we obtain that $\widetilde{\tau_{1}}<t_{m+q}$. Suppose that $\widetilde{\tau_{1}} \in\left[t_{m+k}, t_{m+k+1}\right), 1 \leq$ $k<q$.

We also have two cases. $\left(b_{1 a}\right)$ If $\widetilde{\tau_{1}}$ is not impulsive point, that is to say $\widetilde{\tau_{1}}>t_{m+k}$, then considering the definition of $\widetilde{\tau_{1}}$, we have

$$
V\left(\widetilde{\tau_{1}}, y\left(\widetilde{\tau_{1}}\right)\right)=\frac{\vec{N}}{2} \lambda_{1} \varepsilon^{2} .
$$

By the same argument as the above mentioned, we obtain that (48) still holds.

Hence, from (13), we get

$$
\begin{aligned}
\left(\frac{\vec{N}}{2}+\frac{1}{4}\right) \lambda_{1} \varepsilon^{2} & \leq V\left(\tau_{2}, y\left(\tau_{2}\right)\right) \\
& \leq V\left(\widetilde{\tau_{1}}, y\left(\widetilde{\tau_{1}}\right)\right)+\sum_{i=m+k+1}^{m+q}\left[V\left(t_{i}\right)-V\left(t_{i}^{-}\right)\right] \\
& \leq \frac{\vec{N}}{2} \lambda_{1} \varepsilon^{2}+\sum_{i=m+k+1}^{m+q}\left(\max \left\{\eta_{i}, 1\right\}-1\right) V\left(t_{i}^{-}\right) \\
& \leq \frac{\vec{N}}{2} \lambda_{1} \varepsilon^{2}+\sum_{i=m+k+1}^{m+q}\left(\max \left\{\eta_{i}, 1\right\}-1\right) \lambda_{2} G^{2},
\end{aligned}
$$

which implies that

$$
\frac{1}{4} \lambda_{1} \varepsilon^{2} \leq \sum_{i=m+k+1}^{m+q}\left(\max \left\{\eta_{i}, 1\right\}-1\right) \lambda_{2} G^{2}
$$

which is a contradiction with (29). So $\widetilde{\tau_{1}}$ is some impulsive point.

$\left(b_{1 b}\right)$ If $\widetilde{\tau_{1}}$ is some impulsive point, that is to see $\widetilde{\tau_{1}}=t_{m+k}$, then from the definition of $\widetilde{\tau_{1}}$, it is clear that

$$
V\left({\widetilde{\tau_{1}}}^{-}, y\left({\widetilde{\tau_{1}}}^{-}\right)\right)=V\left(t_{m+k}^{-}, y\left(t_{m+k}^{-}\right)\right)<\frac{\vec{N}}{2} \lambda_{1} \varepsilon^{2}
$$

which implies that

$$
\begin{aligned}
V\left(\widetilde{\tau_{1}}, y\left(\widetilde{\tau_{1}}\right)\right) & =V\left(t_{m+k}, y\left(t_{m+k}\right)\right) \\
& \leq \eta_{m+k} V\left(t_{m+k}^{-}, y\left(t_{m+k}^{-}\right)\right) \\
& <\eta_{m+k} \frac{\vec{N}}{2} \lambda_{1} \varepsilon^{2} \\
& \leq \max \left\{\eta_{m+k}, 1\right\} \frac{\vec{N}}{2} \lambda_{1} \varepsilon^{2} .
\end{aligned}
$$


On the other hand, note that inequality (48) still holds; from (13) and (29), we have

$$
\begin{aligned}
\left(\frac{\vec{N}}{2}+\frac{1}{4}\right) \lambda_{1} \varepsilon^{2} \leq & V\left(\tau_{2}, y\left(\tau_{2}\right)\right) \\
\leq & V\left(\widetilde{\tau_{1}}, y\left(\widetilde{\tau_{1}}\right)\right) \\
& +\sum_{i=m+k+1}^{m+q}\left[V\left(t_{i}\right)-V\left(t_{i}^{-}\right)\right] \\
< & \max \left\{\eta_{m+k}, 1\right\} \frac{\vec{N}}{2} \lambda_{1} \varepsilon^{2} \\
& +\sum_{i=m+k+1}^{m+q}\left(\max \left\{\eta_{i}, 1\right\}-1\right) V\left(t_{i}^{-}\right),
\end{aligned}
$$

which implies that

$$
\begin{aligned}
\frac{1}{4} \lambda_{1} \varepsilon^{2} \leq & \left(\max \left\{\eta_{m+k}, 1\right\}-1\right) \frac{\vec{N}}{2} \lambda_{1} \varepsilon^{2} \\
& +\sum_{i=m+k+1}^{m+q}\left(\max \left\{\eta_{i}, 1\right\}-1\right) \lambda_{2} G^{2} \\
\leq & \frac{1}{6 \vec{N}} \frac{\vec{N}}{2} \lambda_{1} \varepsilon^{2} \\
& +\sum_{i=m+k+1}^{m+q}\left(\max \left\{\eta_{i}, 1\right\}-1\right) \lambda_{2} G^{2} .
\end{aligned}
$$

That means

$$
\frac{1}{6} \lambda_{1} \varepsilon^{2}<\sum_{i=m+k+1}^{m+q}\left(\max \left\{\eta_{i}, 1\right\}-1\right) \lambda_{2} G^{2},
$$

which contradicts (29).

Hence, the first situation $\tau_{2}>t_{m+q}$ is impossible.

$\left(b_{2}\right)$ If $\tau_{2}=t_{m+q}$, then by the same arguments as in the proof in $\left(b_{1}\right)$ and (43), we have

$$
V\left(\tau_{2}^{-}, y\left(\tau_{2}^{-}\right)\right)>\frac{\vec{N}}{2} \lambda_{1} \varepsilon^{2} .
$$

Then let

$$
\begin{aligned}
\widetilde{\tau_{1}}=\inf _{\tau_{1} \leq t<\tau_{2}}\left\{t \mid s \in\left[t, \tau_{2}\right], \frac{\vec{N}}{2} \lambda_{1} \varepsilon^{2}\right. \\
\left.\quad \leq V(s, y(s))<V\left(\tau_{2}, y\left(\tau_{2}\right)\right)\right\} .
\end{aligned}
$$

The rest of the arguments are omitted. Finally we can find our desirable contradiction. Hence, (36) holds.

With above mentioned, the same arguments as before, if we replace $T_{1}$ with $N^{\star}$, then there exists a $T_{2}=T_{1}+w_{1}=$ $N^{\star}+2 w_{1}$ such that for $t>T_{2}$,

$$
V(t, y(t))<\frac{\vec{N}}{2} \lambda_{1} \varepsilon^{2}
$$

Let $T_{2}$ replace $T_{1}$; then there exists a $T_{3}=N^{\star}+3 w_{1}$ such that for $t>T_{3}$,

$$
V(t, y(t))<\left(\frac{\vec{N}}{2}-\frac{1}{4}\right) \lambda_{1} \varepsilon^{2} .
$$

By induction hypothesis, we may prove, in general, that there exists a $T_{2 \vec{N}}=N^{\star}+2 \vec{N} w_{1}$ such that $t>T_{2 \overleftarrow{N}}$,

$$
V(t, y(t))<\left(\frac{\vec{N}}{2}-\frac{\vec{N}-2}{2}\right) \lambda_{1} \varepsilon^{2}=\lambda_{1} \varepsilon^{2} .
$$

Therefore, we obtain that $\|y(t)\|<\varepsilon, t>N^{\star}+2 N w_{1}$. In view of the choice of $N^{\star}, \vec{N}$, and $w_{1}$, the zero solution of (4) is uniformly asymptotically stable; that is, the equilibrium point of (1) is uniformly asymptotically stable. The proof of Theorem 7 is therefore complete.

Let $\left|1+d_{k}^{(i)}\right| \neq\left|1+d_{k}^{(j)}\right|, c_{i} \neq c_{j}, i \neq j, i, j \in \Lambda, k=1,2, \ldots$, in Theorem 7; then we can have the following result.

Corollary 8. The equilibrium point of system (1) is uniformly asymptotically stable, if there exists $n \times n$ symmetric and positive definite matrix $P$ satisfying

$\left(I_{1}\right) \bar{\eta} \doteq \prod_{k=1}^{\infty} \max \left\{\max _{i \in \Lambda}\left(1+d_{k}^{(i)}\right)^{2}, 1\right\}<\infty ;$

$\left(I_{2}\right) \lambda_{3}<(-M-N) / \lambda_{1}$, where $\lambda_{1}$ is the smallest eigenvalue of $P$ and $\lambda_{3}$ is the largest eigenvalue of $2 C+A A^{T} P+$ $B B^{T} P$.

Remark 9. For using the less conservative conditions in Theorem 7, our results obviously improve some results established in the earlier references. In [5], condition $y\left(t_{k}\right)=$ $D y\left(t_{k}^{-}\right)$holds for all $k \in \mathbb{Z}_{+}$; here note in our Theorem 7 that we only require that the solutions satisfy the hypothesis $\left(H_{1}\right)$ at impulsive points. In addition, our conditions are without requirement of the range of the largest eigenvalues of $P^{-1} D^{T} P D$ on $(0,1)$, which are milder than the restrictions in [5].

By utilizing Lemma 6, we will give some sufficient conditions for globally exponential stability of the equilibrium point of system (1).

Theorem 10. Assume that there exists $n \times n$ symmetric and positive definite matrix $P$ such that $\lambda_{1}>0$ is the smallest eigenvalue of $P, \lambda_{3}$ is the largest eigenvalue of $P^{-1}(C P+P C+$ $\left.P A A^{T} P+P B B^{T} P\right), \eta_{k}$ is the largest eigenvalue of $P^{-1} D_{k} P D_{k}$, and $\lambda_{1}, \lambda_{3}$ and $\eta_{k}$ satisfy the following conditions:

$\left(H_{3}\right)$ one has

$$
\lambda_{3}+\frac{M}{\lambda_{1}}+\frac{N}{\lambda_{1}} \cdot \max _{k \in \mathbb{Z}_{+}}\left\{\frac{1}{\eta_{k}}, 1\right\}<0,
$$

$\left(H_{4}\right)$ there exist constants $\mathbb{U}(>0), \delta(\geq 0)$ such that $\delta<\mu$ and the following inequality

$$
\sum_{k=1}^{m} \ln \eta_{k}-\delta\left(t_{m}-t_{0}\right)<\mathbb{U} \quad \forall m \in \mathbb{Z}_{+} \text {holds },
$$


where $\mu$ satisfies the following inequality:

$$
0<\mu \leq-\lambda_{3}-\frac{M}{\lambda_{1}}-\frac{N}{\lambda_{1}} \max _{k \in \mathbb{Z}_{+}}\left\{\frac{1}{\eta_{k}}, 1\right\} \cdot e^{\mu \tau} .
$$

Then the equilibrium point of the system (1) is globally exponentially stable, and the exponential convergence rate is equal to $(\mu-\delta) / 2$.

Proof. Let $y(t)=y(\sigma, \varphi)(t)$ be a solution of (4) through $(\sigma, \varphi), \sigma \geq t_{0}$ (for convenience, we assume that $\sigma=t_{0}$ ). We next will prove that the zero solution of (4) is globally exponentially stable. Construct the Lyapunov functional as in Theorem 7; that is, $V(t, y(t))=y^{T}(t) P y(t)$; then we obtain the following:

(1) $\lambda_{1}\|y(t)\|^{2} \leq V(t, y(t)) \leq \lambda_{2}\|y(t)\|^{2}$, where $\lambda_{2}$ is the largest eigenvalue of $P$;

(2) for $t \in\left[t_{k}, t_{k+1}\right), k=1,2, \ldots$,

$$
\begin{aligned}
\left.D^{+} V(t, y(t))\right|_{(4)} \leq & {\left[\lambda_{3}+M \lambda_{1}^{-1}\right] y^{T}(t) P y(t) } \\
& +N \lambda_{1}^{-1} y_{\tau}^{T} P y_{\tau} ;
\end{aligned}
$$

(3) $V\left(t_{k}, y\left(t_{k}\right)\right) \leq \eta_{k} V\left(t_{k}^{-}, y\left(t_{k}^{-}\right)\right)$.

From (2), we have for $t \in\left[t_{k}, t_{k+1}\right), k=1,2, \ldots$,

$$
\left.D^{+} V(t, y(t))\right|_{(4)} \leq-P V(t, y(t))+Q \widetilde{V}(t, y(t))
$$

where $\widetilde{V}(t)=\sup _{t-\tau \leq s \leq t} V(s), P=-\lambda_{3}-M \lambda_{1}^{-1}$, and $Q=$ $N \lambda_{1}^{-1}$.

For any $t \geq t_{0}$, suppose that $t \in\left[t_{m}, t_{m+1}\right), m \geq 0$. By Lemma 6 and condition $\left(H_{4}\right)$, we obtain

$$
\begin{aligned}
V(t) & \leq \widetilde{V}\left(t_{0}\right)\left(\prod_{k=1}^{m} \eta_{k}\right) e^{-\mu\left(t-t_{0}\right)} \\
& \leq \widetilde{V}\left(t_{0}\right) e^{\mathbb{U}} \cdot e^{\delta\left(t_{m}-t_{0}\right)} \cdot e^{-\mu\left(t-t_{0}\right)} \\
& \leq \widetilde{V}\left(t_{0}\right) e^{\mathbb{U}} \cdot e^{\delta\left(t-t_{0}\right)} \cdot e^{-\mu\left(t-t_{0}\right)} \\
& \leq \lambda_{2} e^{\mathbb{U}}\|\varphi\|_{\tau}^{2} e^{-(\mu-\delta)\left(t-t_{0}\right)},
\end{aligned}
$$

where $\mu$ satisfies inequality (66).

Hence, we obtain for any $t \geq t_{0}$,

$$
\|y(t)\| \leq e^{(1 / 2) \mathbb{U}} \sqrt{\frac{\lambda_{2}}{\lambda_{1}}}\|\varphi\|_{\tau} e^{-((\mu-\delta) / 2)\left(t-t_{0}\right)}, \quad t \geq t_{0},
$$

where $\mu$ satisfies inequality (66).

Therefore, the zero solution of system (4) is globally exponentially stable; that is, the equilibrium point of system (1) is globally exponentially stable. The proof of Theorem 10 is complete.

Remark 11. In Theorem 10, if $\sup _{n \in \mathbb{Z}_{+}}\left(\prod_{k=1}^{n} \eta_{k}\right)<\infty$, then we can choose $\delta=0$ in condition $\left(\mathrm{H}_{3}\right)$.

Let $\eta_{k} \in(0,1], k=1,2, \ldots$ in Theorem 7 ; then we can have the following result.
Corollary 12. Assume that there exists $n \times n$ symmetric and positive definite matrix $P$ such that $\lambda_{1}$ is the smallest eigenvalue of $P, \lambda_{3}$ is the largest eigenvalue of $P^{-1}\left(C P+P C+P A A^{T} P+\right.$ $\left.P B B^{T} P\right), \eta_{k}$ is the largest eigenvalue of $P^{-1} D_{k} P D_{k}$, and condition

$$
\lambda_{1} \lambda_{3}+M+N<0 \text { holds. }
$$

Then the equilibrium point of the system (1) is globally exponentially stable, and the exponential convergence rate is equal to $\mu / 2$, where $\mu$ satisfies the following inequality:

$$
0<\mu \leq-\lambda_{3}-\frac{M}{\lambda_{1}}-\frac{N}{\lambda_{1}} \max _{k \in \mathbb{Z}_{+}} \eta_{k} \cdot e^{\mu \tau} .
$$

\section{Example}

In this section, we present a numerical example to illustrate our results.

Example 1. We consider Example 1 in [2] as follows:

$$
\begin{aligned}
u_{1}^{\prime}(t)= & -2.5 u_{1}(t)-0.5 f\left(u_{1}(t)\right) \\
& +0.1 f\left(u_{2}(t)\right)-0.1 f\left(u_{1}(t-\tau)\right) \\
& +0.2 f\left(u_{2}(t-\tau)\right)-1, \\
u_{2}^{\prime}(t)= & -2 u_{1}(t)+0.2 f\left(u_{1}(t)\right) \\
& -0.1 f\left(u_{2}(t)\right)+0.2 f\left(u_{1}(t-\tau)\right) \\
& +0.1 f\left(u_{2}(t-\tau)\right)+4,
\end{aligned}
$$

with impulses

$$
\begin{gathered}
d_{k}^{(1)}=\sqrt{1+\frac{1}{2 k^{2}}}-1, \\
d_{k}^{(2)}=\sqrt{1+\frac{1}{k^{2}}}-1, \quad t_{k}=k, k \in \mathbb{Z}_{+} .
\end{gathered}
$$

The delayed feedback matrixes $A, B$, and $C$ are

$$
\begin{gathered}
C=\left(\begin{array}{cc}
-2.5 & 0 \\
0 & -2
\end{array}\right), \quad A=\left(\begin{array}{cc}
-0.5 & 0.1 \\
0.2 & -0.1
\end{array}\right), \\
B=\left(\begin{array}{cc}
-0.1 & 0.2 \\
0.2 & 0.1
\end{array}\right) .
\end{gathered}
$$

Since the activation function in [2] is described by $f=f_{i}=$ $0.5(|x+1|-|x-1|), i=1,2$, then we have $M=N=1$. We may choose $P=E$ (unit matrix); then $\lambda_{1}=\lambda_{2}=1$. Note that

$$
\begin{gathered}
P^{-1} D_{k} P D_{k}=D_{k}^{2}=\left(\begin{array}{cc}
1+\frac{1}{2 k^{2}} & 0 \\
0 & 1+\frac{1}{k^{2}}
\end{array}\right), \\
P^{-1}\left(C P+P C+P A A^{T} P+P B B^{T} P\right) \\
=2 C+A A^{T}+B B^{T} \\
=\left(\begin{array}{cc}
-4.69 & -0.11 \\
-0.11 & -3.9
\end{array}\right)=\Delta .
\end{gathered}
$$




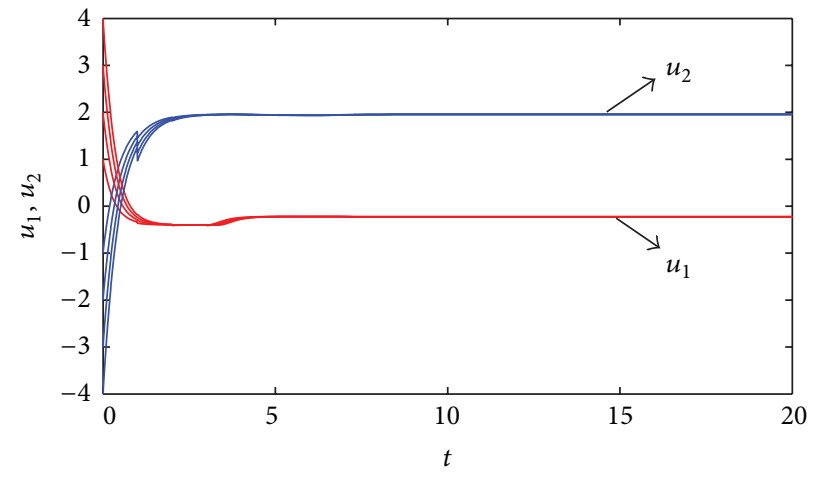

(a)

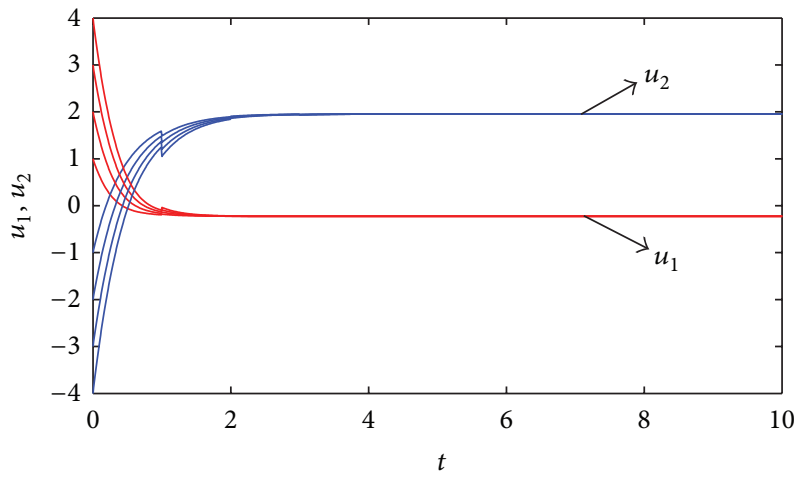

(b)

FIGURE 1: (a) State trajectories of system (73) with impulses (74) and $\tau=3$. (b) State trajectories of system (73) with impulses (79) and $\tau=0.17$.

then we get $\eta_{k}=1+1 / k^{2}, \bar{\eta}=\prod_{k=1}^{\infty}\left(1+1 / k^{2}\right)<\infty$. Matrix $\Delta$ 's characteristic equation is

$$
\lambda^{2}+8.59 \lambda+18.2789=0 .
$$

By a straightforward calculation, we obtain that the largest characteristic root $\lambda_{3} \approx-3.885<-2$. By Theorem 7 , the equilibrium point of system (73) is uniformly asymptotically stable with impulses (74) for any $\tau>0$, which is shown in Figure 1(a).

However, the criteria in [5] are invalid here. In fact, condition $y\left(t_{k}\right)=D y\left(t_{k}^{-}\right)$is not satisfied here. Moreover, because of the impulsive effect, the criteria in [2] are also invalid here. Therefore, our results are less conservative than those given result in $[2,5]$.

Furthermore, let $\tau=0.17$. Note that $\prod_{k=1}^{\infty}\left(1+1 / k^{2}\right)<$ $\infty, \lambda_{3}=-3.885<-2$; then one can choose $\delta=0$ in Theorem 10. So all conditions of Theorem 10 are satisfied. Therefore, the equilibrium point of system (73) is globally exponentially stable, and the exponential convergence rate is equal to $\mu$, where $\mu>0$ satisfies

$$
\mu+e^{0.17 \mu}-2.885<0 .
$$

If $d_{k}^{(1)}, d_{k}^{(2)}$ in above example are given as follows:

$$
\begin{gathered}
d_{k}^{(1)}= \begin{cases}\sqrt{2.2}-1, & k=2 n-1, \\
\sqrt{0.23}-1, & k=2 n, n \in \mathbb{Z}_{+},\end{cases} \\
d_{k}^{(2)} \begin{cases}\sqrt{1.7}-1, & k=2 n-1, \\
\sqrt{0.4}-1, & k=2 n, n \in \mathbb{Z}_{+},\end{cases}
\end{gathered}
$$

then we finally get $\lambda_{3}=-3.885<-3.500, \delta=0$. So all conditions of Theorem 10 are still satisfied. Therefore, the equilibrium point of system (73) is globally exponentially stable with impulses (79), and the exponential convergence rate is equal to $\mu$, where $\mu>0$ satisfies

$$
\mu+2.5 e^{0.17 \mu}-2.885<0 .
$$

The simulation is shown in Figure 1(b). However, it is easy to check that the impulsive delayed Halanay inequality in [23, $26]$ is not feasible here. Thus our results can be applied to the case not covered in $[23,26]$.

\section{Conclusion}

The uniform asymptotic stability and global exponential stability of impulsive HNN with time delays are considered in this paper. Some new stability conditions are obtained by means of constructing the extended impulsive Halanay inequality, Lyapunov functional methods, and linear matrix inequality approach. Moreover, our results can be applied to the case not covered in some other existing criteria. Hence, the results extend and improve the earlier publications. An example is given to illustrate the feasibility of the results and the effects of impulses.

\section{Acknowledgments}

This work was jointly supported by the Project of Shandong Province Higher Educational Science and Technology Program (J12LI04), Research Fund for Excellent Young and Middle-Aged Scientists of Shandong Province (BS2012DX039), and National Natural Science Foundation of China (11226136, 11301308).

\section{References}

[1] Q. Zhang, X. Wei, and J. Xu, "Delay-dependent global stability condition for delayed Hopfield neural networks," Nonlinear Analysis: Real World Applications, vol. 8, no. 3, pp. 997-1002, 2007.

[2] Q. Zhang, X. Wei, and J. Xu, "Delay-dependent global stability results for delayed Hopfield neural networks," Chaos, Solitons \& Fractals, vol. 34, no. 2, pp. 662-668, 2007.

[3] R. Rakkiyappan and P. Balasubramaniam, "On exponential stability results for fuzzy impulsive neural networks," Fuzzy Sets and Systems, vol. 161, no. 13, pp. 1823-1835, 2010.

[4] X. Liao and D. Xiao, "Globally exponential stability of Hopfield neural networks with time varying delays," Acta Electronica Sinica, vol. 28, no. 4, pp. 87-90, 2000.

[5] Y. Zhang and J. Sun, "Stability of impulsive neural networks with time delays," Physics Letters A, vol. 348, no. 1-2, pp. 44-50, 2005.

[6] Z. C. Yang and D. Y. Xu, "Global exponential stability of Hopfield neural networks with variable delays and impulsive 
effects," Applied Mathematics and Mechanics, vol. 27, pp. 1517$1522,2006$.

[7] X. Li and X. Fu, "Global asymptotic stability of stochastic Cohen-Grossberg-type BAM neural networks with mixed delays: an LMI approach," Journal of Computational and Applied Mathematics, vol. 235, no. 12, pp. 3385-3394, 2011.

[8] X. Li, "Global robust stability for stochastic interval neural networks with continuously distributed delays of neutral type," Applied Mathematics and Computation, vol. 215, no. 12, pp. 4370-4384, 2010.

[9] Y. Xia, Z. Yang, and M. Han, "Synchronization schemes for coupled identical Yang-Yang type fuzzy cellular neural networks," Communications in Nonlinear Science and Numerical Simulation, vol. 14, no. 9-10, pp. 3645-3659, 2009.

[10] D. D. Bainnov and P. S. Simeonov, Systems with Impulsive Effect Stability Theory and Applications, Ellis Horwood, New York, NY, USA, 1989.

[11] X. Fu, B. Yan, and Y. Liu, Introduction of Impulsive Differential Systems, Science Press, Beijing, China, 2005.

[12] Y. Xia, "Impulsive effect on the delayed cohen-grossberg-type BAM neural networks," Neurocomputing, vol. 73, no. 13-15, pp. 2754-2764, 2010.

[13] Y. Xia and P. J. Y. Wong, "Global exponential stability of a class of retarded impulsive differential equations with applications," Chaos, Solitons \& Fractals, vol. 39, no. 1, pp. 440-453, 2009.

[14] A. O. Ignatyev, "On the stability of invariant sets of systems with impulse effect," Nonlinear Analysis: Theory, Methods \& Applications $A$, vol. 69, no. 1, pp. 53-72, 2008.

[15] Z. Guan and G. Chen, "On delayed impulsive Hopfield neural networks," Neural Networks, vol. 12, no. 2, pp. 273-280, 1999.

[16] Z. Guan, J. Lam, and G. Chen, "On impulsive autoassociative neural networks," Neural Networks, vol. 13, no. 1, pp. 63-69, 2000.

[17] R. Rakkiyappan, P. Balasubramaniam, and J. Cao, "Global exponential stability results for neutral-type impulsive neural networks," Nonlinear Analysis: Real World Applications, vol. 11, no. 1, pp. 122-130, 2010.

[18] X. Li and R. Rakkiyappan, "Impulse controller design for exponential synchronization of chaotic neural networks with mixed delays," Communications in Nonlinear Science and Numerical Simulation, vol. 18, no. 6, pp. 1515-1523, 2013.

[19] X. Liu, K. L. Teo, and B. Xu, "Exponential stability of impulsive high-order Hopfield-type neural networks with time-varying delays," IEEE Transactions on Neural Networks, vol. 16, no. 6, pp. 1329-1339, 2005.

[20] S. Mohamad, K. Gopalsamy, and H. Akça, "Exponential stability of artificial neural networks with distributed delays and large impulses," Nonlinear Analysis: Real World Applications, vol. 9, no. 3, pp. 872-888, 2008.

[21] H. Gu, "Mean square exponential stability in high-order stochastic impulsive BAM neural networks with time-varying delays," Neurocomputing, vol. 74, no. 5, pp. 720-729, 2011.

[22] Y. Zhang, "Robust exponential stability of uncertain impulsive neural networks with time-varying delays and delayed impulses," Neurocomputing, vol. 74, no. 17, pp. 3268-3276, 2011.

[23] Z. Yang and D. Xu, "Stability analysis of delay neural networks with impulsive effects," IEEE Transactions on Circuits and Systems I, vol. 52, pp. 517-521, 2005.

[24] X. Liao, G. Chen, and E. N. Sanchez, "LMI-based approach for asymptotically stability analysis of delayed neural networks," IEEE Transactions on Circuits and Systems I, vol. 49, no. 7, pp. 1033-1039, 2002.
[25] X. Fu and X. Li, "Global exponential stability and global attractivity of impulsive Hopfield neural networks with time delays," Journal of Computational and Applied Mathematics, vol. 231, no. 1, pp. 187-199, 2009.

[26] J. Zhou, L. Xiang, and Z. Liu, "Synchronization in complex delayed dynamical networks with impulsive effects," Physica A, vol. 384, no. 2, pp. 684-692, 2007. 


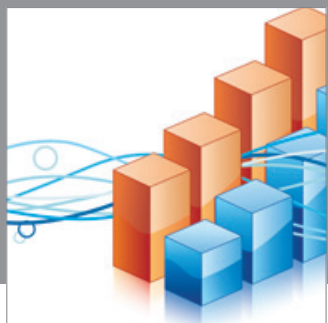

Advances in

Operations Research

mansans

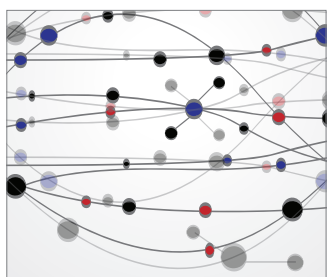

The Scientific World Journal
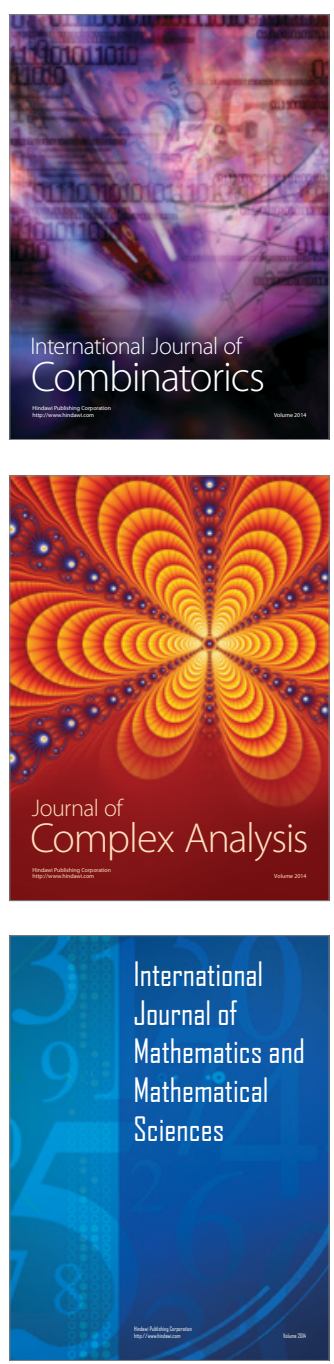
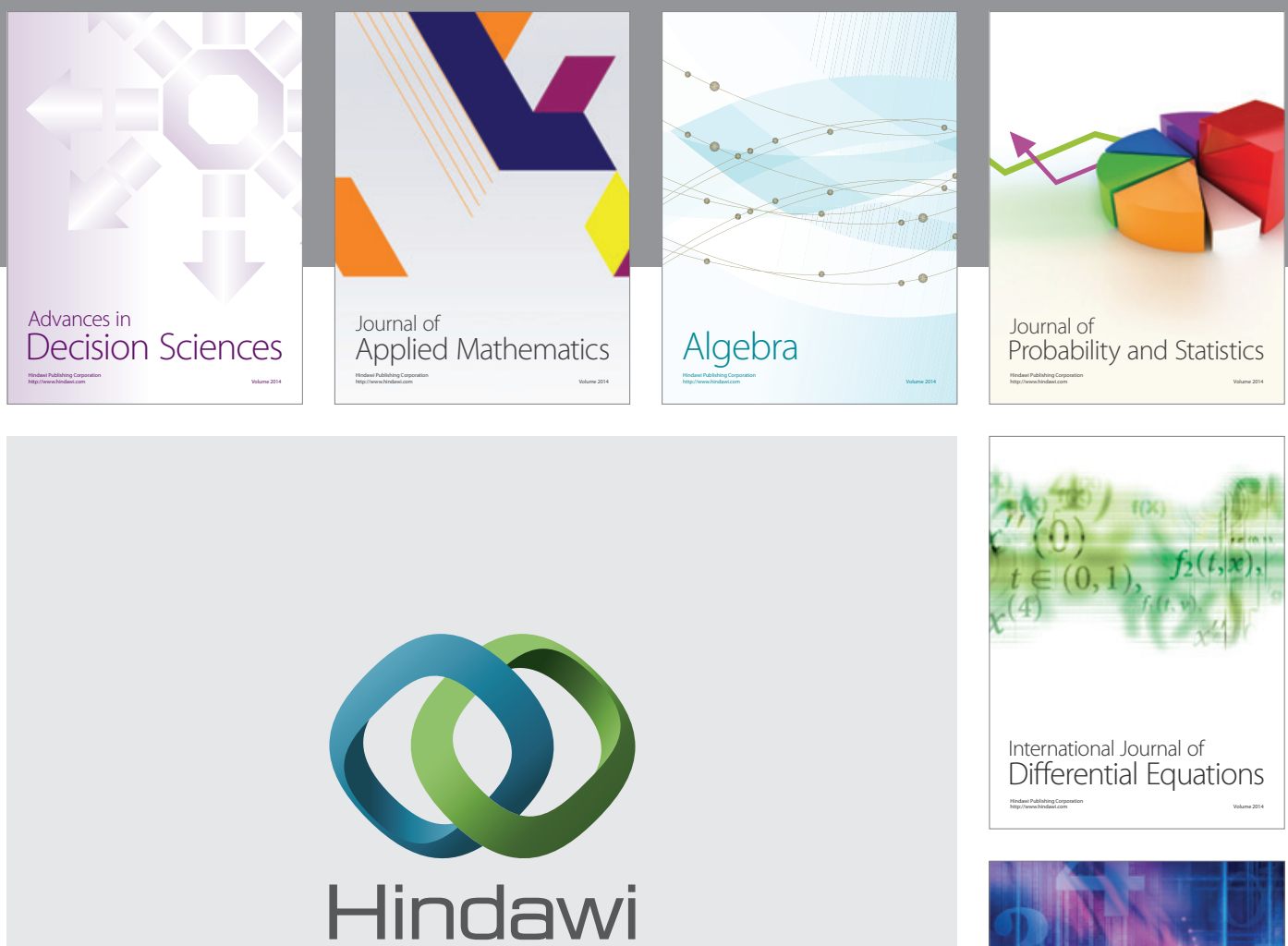

Submit your manuscripts at http://www.hindawi.com
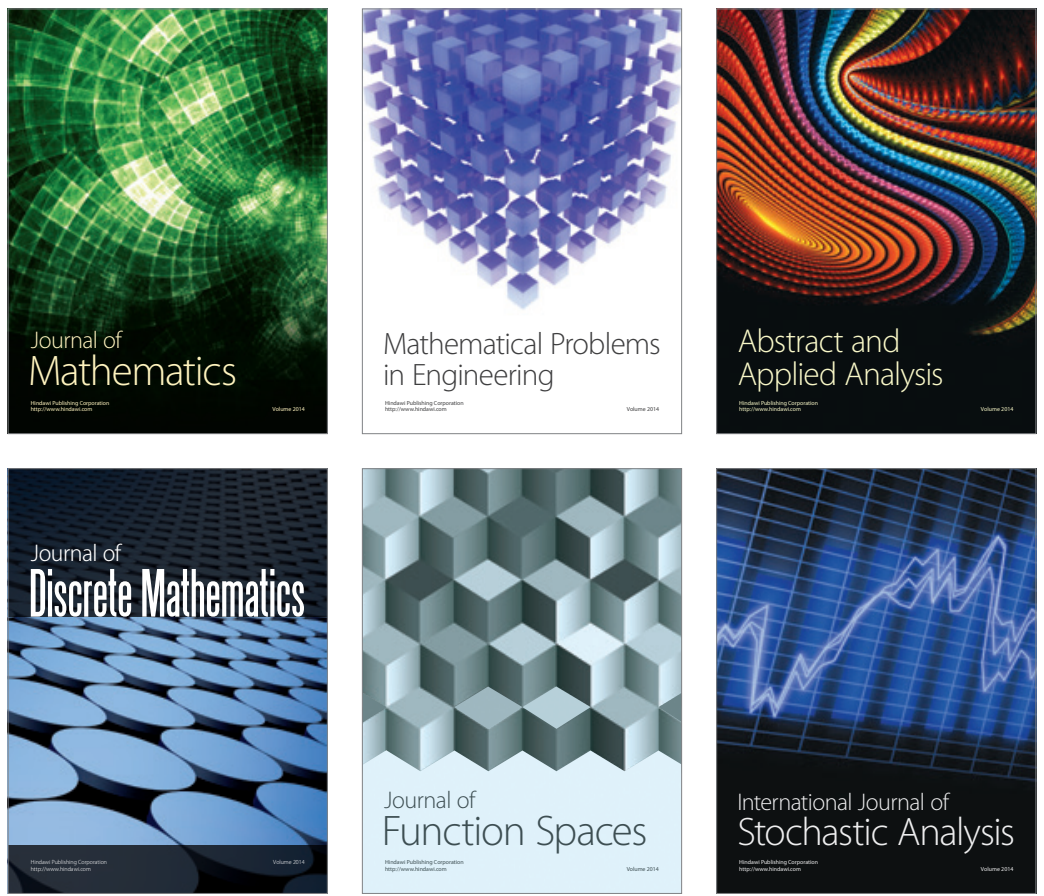

Journal of

Function Spaces

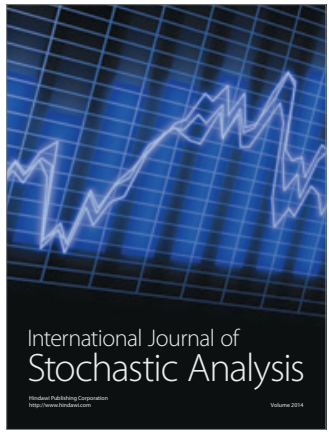

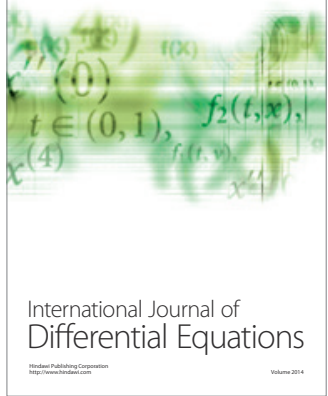
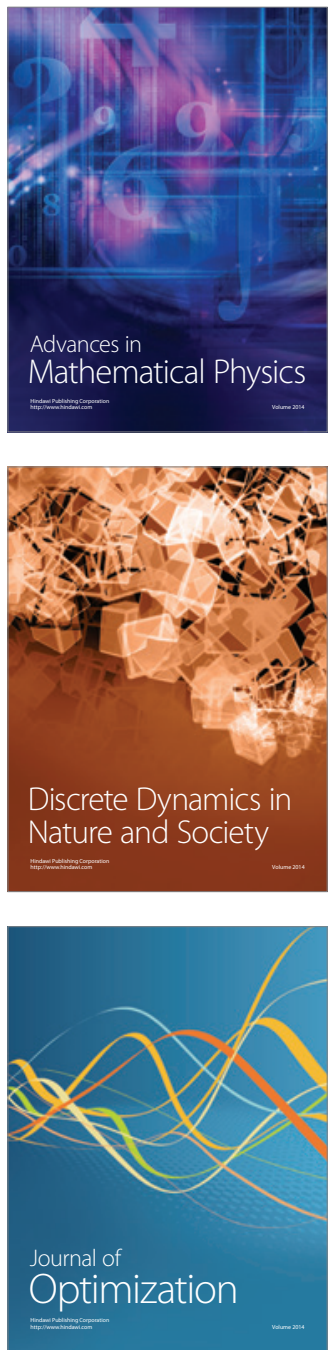